\title{
Rice OsHSFA3 Gene Improves Drought Tolerance by Modulating Polyamine Biosynthesis Depending on Abscisic Acid and ROS Levels
}

\author{
Ming-Dong Zhu ${ }^{1,+}{ }^{\oplus}$, Meng Zhang ${ }^{2,+}$, Du-Juan Gao ${ }^{1}$, Kun Zhou ${ }^{1}$, Shan-Jun Tang ${ }^{1}$, Bin Zhou ${ }^{1, *}$ \\ and Yan-Mei Lv ${ }^{1, *}$ \\ 1 Hunan Rice Research Institute, Key Laboratory of Indica Rice Genetics and Breeding in the Middle and \\ Lower Reaches of Yangtze River Valley, Ministry of Agriculture, Changsha 410125, China; \\ uhz_uhz@hotmail.com (M.-D.Z.); gaodujuan221@163.com (D.-J.G.); zhzhkp@hotmail.com (K.Z.); \\ shanjunt@126.com (S.-J.T.) \\ 2 Hunan Key Laboratory of Plant Functional Genomics and Developmental Regulation, College of Biology, \\ Hunan University, Changsha 410082, China; zhangmeng2019@hnu.edu.cn \\ * Correspondence: zhoubin750517@163.com (B.Z.); lym3628901@163.com (Y.-M.L.) \\ + These authors contributed equally to this work.
}

Received: 10 February 2020; Accepted: 7 March 2020; Published: 9 March 2020

\begin{abstract}
Drought is a serious problem, which causes heavy yield losses for rice. Heat-shock factors (HSFs) had been implicated in tolerance to drought and high temperature. However, there has not been much functional characterization and mechanism clarification in rice. Previously, we found an HSF gene, OsHSFA3, was highly related with drought tolerance after screening from 10,000 different samples. Herein, we cloned the OSHSFA3 from rice and overexpressed it in Arabidopsis thaliana to study its regulatory mechanism of drought tolerance. Phenotypic and physiological assays of the transgenic Arabidopsis lines showed that overexpression of OsHSFA3 confers drought tolerance by reducing water loss and reactive oxygen species (ROS) levels, whereas it increases abscisic acid (ABA) levels. However, enzymatic antioxidants such as activity levels of superoxide dismutase, peroxidase and catalase were not significantly different between wild type and transgenic lines. Instead, we observed a significant increase in polyamine content, which was correlated with increased AtADC1, AtADC2, SPDS1 and SPMS expression levels. In silico and in vivo analyses confirmed that OsHSFA3 is a nuclear-localized gene. In addition, OsHSFA3 can bind to the promoter of AtADC1 and OsADC via a yeast one-hybrid assay. Overall, this study reveals that OsHSFA3 improves drought tolerance in Arabidopsis not only by increasing ABA levels, but also by modulating polyamine levels to maintain ROS homeostasis, therefore it could be a strong candidate to develop drought-tolerant rice cultivars.
\end{abstract}

Keywords: drought; abscisic acid; gene expression; polyamines; reactive oxygen species

\section{Introduction}

Abiotic stresses such as drought, salinity and suboptimal temperature are serious threats for agriculture and global food security [1,2]. These stresses negatively affect plant growth and development and ultimately result in poor crop production [3-5]. Rice is a major grain crop and feeds about half of the world population [6,7]. Drought is one of the major abiotic stresses negatively influencing rice grain yield $[8,9]$. It causes osmotic stress to plants and leads to over-accumulation of reactive oxygen species (ROS) in different cellular compartments [10]. This over-accumulation of ROS causes oxidative stress to plants and damages various cellular organelles including the cell membrane [11,12]. It also leads to early senescence of leaves and other tissues via enhanced programmed cell death [11,13]. 
Since plants cannot move from one place to other to avoid the harmful effects of stresses, they adopt various mechanisms for stress tolerance. The antioxidant defense system is one of the major tolerance mechanisms for ROS scavenging under abiotic stresses [14]. Enzymatic antioxidants including superoxide dismutase (SOD), peroxidase (POD) and catalase (CAT) are key ROS-scavenging enzymes $[15,16]$. Similarly, polyamines are vital plant metabolites that serve as important ROS scavengers and also induce stomatal closure under drought stress $[17,18]$. The enzyme arginine decarboxylase (ADC) catalyzes arginine to agmatine, which is the precursor of putrescine and higher polyamines (PAs), such as spermidine and spermine. Several genes have been reported to control the production of polyamines in plants [18,19]. In Arabidopsis thaliana, ADC only has two paralogues, $A t A D C 1$ and $A t A D C 2$ [20]. In rice, several genes regulate the biosynthesis of polyamines in a collective way and contribute to drought tolerance [21,22]. Thus, exploiting such tolerance mechanisms provide an amazing option to cope with drought stress in rice.

Transcription factors (TFs) are well-known for their role in abiotic stress tolerance in plants $[8,23]$. Among the different TF families, heat shock factors (HSFs), WRKY-motif containing proteins (WRKY), myeloblastosis (MYB) and APETALA2/ethylene response factor (AP2/ERF) families are the most promising for their role in drought and other abiotic stress tolerance [18,24-27]. HSF is a large gene family in rice with 25 members and several members of this family have been characterized for their role in stress tolerance $[28,29]$. Abscisic acid (ABA) and ROS are important signaling molecules and regulate the expression of various stress responsive genes [16,30,31]. In rice, expression levels of HSF genes OsHsfA4a and OsHsfA2 were upregulated under ABA and ROS treatments and thus regulate stress tolerance [32]. Overexpression of rice $\mathrm{OsHsfC1b}$ in Arabidopsis causes salinity tolerance in transgenic plants [33]. Similarly, overexpression of rice OsHsfA2e in Arabidopsis enhanced tolerance against multiple abiotic stresses [34].

Previously, we observed drought-resistant lines from more than 10,000 rice cultivars, and finally we found that OsHSFA3 might be the core drought-responsive gene in a drought-tolerant cultivar 'Hunan' [35]. To further evaluate the function of OsHSFA3 and clarify its mechanism in drought tolerance, we applied the model plant Arabidopsis thaliana for better understanding. Finally, our results demonstrated that OsHSFA3 imparts drought tolerance by regulating ROS and polyamine biosynthesis levels.

\section{Results}

\subsection{OsHSFA3 Is a Drought-Responsive Gene and Highly Accumulated in Shoot}

Previous transcriptome analysis, gene coexpression analysis and genome-wide studies have identified a wide range of heat shock factors (HSFs) in the rice genome [28,36]. Of these rice HSFs, OsHSFA3 was reported to be responsive to cold and drought stress [35,36]. The homologous gene of OsHSFA3 in Arabidopsis thaliana (AT5G03720, AtHSFA3) is quite different in terms of gene structure (two introns and three exons) and protein similarity ( $40 \%$ identity), indicating that OsHSFA 3 and AtHSFA3 may have distinct functional properties. However, its functional characterization has not been reported yet. Here we performed a detailed functional characterization of OSHSFA3 to understand its molecular mechanism for drought tolerance.

To confirm if OSHSFA3 is responsive to drought stress, we measured mRNA abundance of OSHSFA3 under different drought stress durations using real-time quantitative PCR (qRT-PCR). OsHSFA3 mRNA accumulated in rice within two days of drought treatment and increased many fold in a time-dependent manner (Figure 1A). After four days of drought stress, the relative expression showed a two-fold increase as compared to the relative expression after two days under stress. Further extension of drought stress duration to seven days resulted in higher relative expression, indicating that OsHSFA3 is a drought-responsive gene (Figure 1A). We then examined the tissue-specific expression pattern of OsHSFA3 in four rice tissues, i.e., leaf, shoot, root and seed. OsHSFA3 was expressed in all four organs, however, the highest relative expression was observed in shoot followed by leaf, root and 
seed (Figure 1B). The relative expression levels in root and seed were relatively lower than in the other organs.

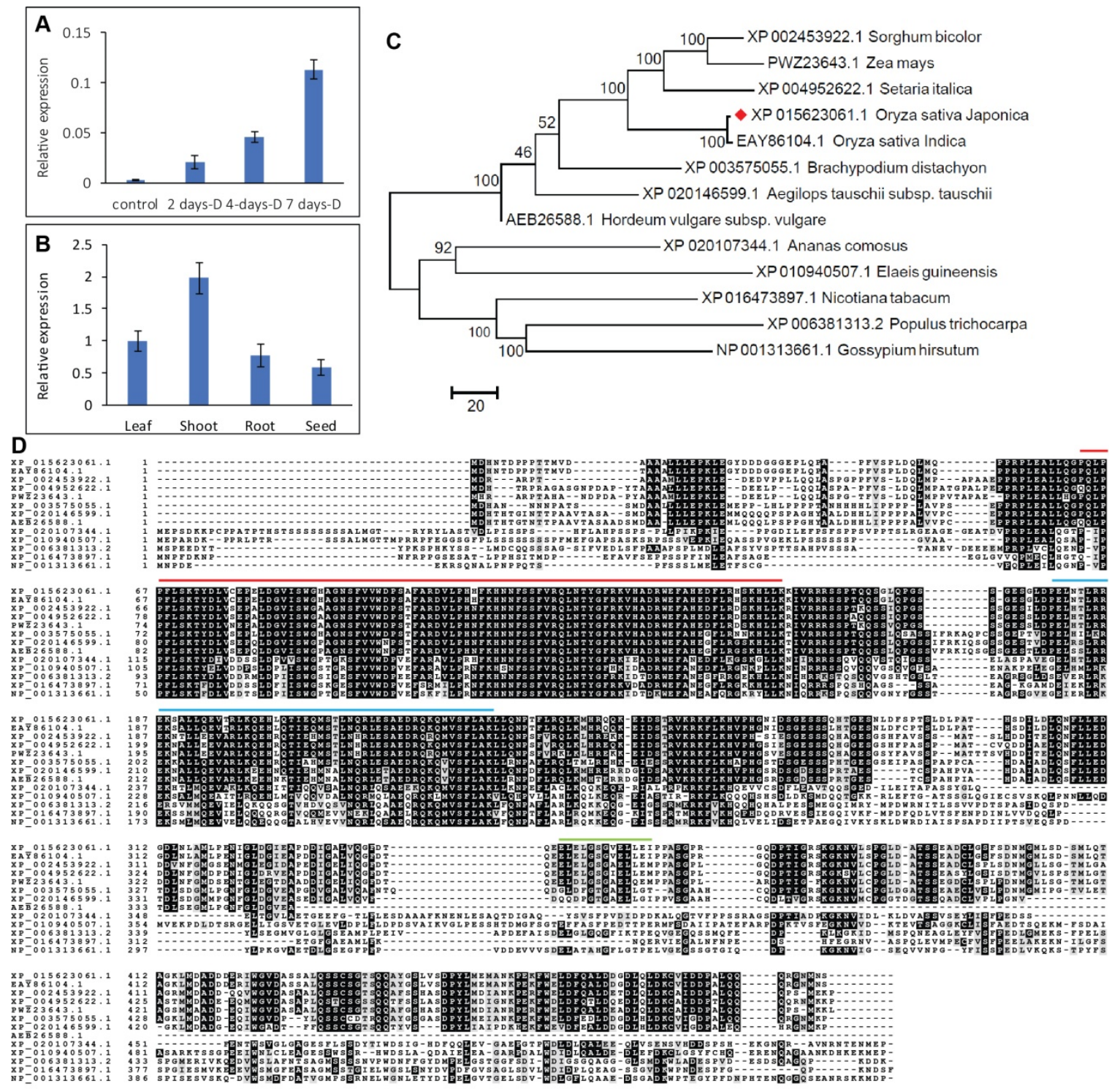

Figure 1. Expression pattern and sequence analysis of OsHSFA3. (A) Relative expression of OsHSFA3 under control and time course drought treatment in rice. (B) Relative expression of OsHSFA3 in different rice tissues. (C) Maximum likelihood phylogenetic tree based on amino acid sequences of OsHSFA3 and orthologs from different species. Red rhombus is the OsHSF3. (D) Multiple sequence alignment showing heat-shock factor (HSF) domain (64-157 aa, under red bar), coiled-coil domain (180-227 aa, under blue bar), and a low-complexity domain (345-357 aa, under green bar).

\subsection{Structural Features of OsHSFA3 and Evolutionary Relationship}

The OsHSFA3 gene sequence was obtained from the Rice genome annotation project (http://rice. plantbiology.msu.edu/) under gene locus LOC_Os02g32590. The OsHSFA3 gene located on chromosome 2 of rice has a full-length CDS (coding sequence) of $1497 \mathrm{bp}$ which encodes a polypeptide of 498 amino acids, consisting of one HSF domain (64-157 aa), one low-complexity domain (15-21 aa), a coiled-coil domain (180-227 aa), and a low-complexity domain (345-357 aa) (Figure S2A). Gene structure analysis indicated that the OsHSFA3 gene has one intron of $1298 \mathrm{bp}$ and two exons (Figure S2B). To investigate the evolutionary relationship of OsHSFA3 with its homologs from different plant species, a phylogenetic 
tree was constructed using orthologues of OsHSFA3 proteins from 13 plant species. The phylogenetic analysis divided the OsHSFA3 orthologues into two main groups, that is, monocots and dicots. Logically, OsHSFA3 falls into the group of monocots suggesting that it is closely related to the members of HSFs in monocots; particularly grouped with HSFs of Setariaitalica, Zea mays and Sorghum bicolor (Figure 1C). We then performed alignment of these protein sequences using ClustalW to identify the conserved regions among different species. The alignment demonstrated that regions containing an HSF domain (64-157 aa), coiled-coil domain (180-227 aa), and a low-complexity domain (345-357 aa) were highly conserved among all species (Figure 1D). However, the other protein sequence is mostly conserved among the monocots, while considerable variation is present with dicots (Figure 1D).

\subsection{OsHSFA3 Localized in Nucleus}

To see the subcellular localization of OsHSFA3, we first predicted its localization using bioinformatics tools. The online tool WoLF PSORT predicted its subcellular localization in the nucleus. To verify the subcellular localization, the full-length OsHSFA3 CDS without the termination codon was fused with the green fluorescence protein (GFP) coding sequence in vector pBWA(V)HS-GLosGFP to express the OsHSFA3-GFP recombinant protein. The constructed plasmid was then transformed into Arabidopsis protoplasts and observed under a confocal laser microscope. The GFP signals were colocalized with nuclear marker signals and merged well, which confirmed that OsHSPA3 was localized in the nucleus (Figure 2A-E). We also transformed a control plasmid containing only the GFP sequence without OsHSFA3 into Arabidopsis protoplasts. As expected, this construct displayed GFP signals in all parts of cell, not only in nucleus, proving the reliability of our results (Figure 2F-I).
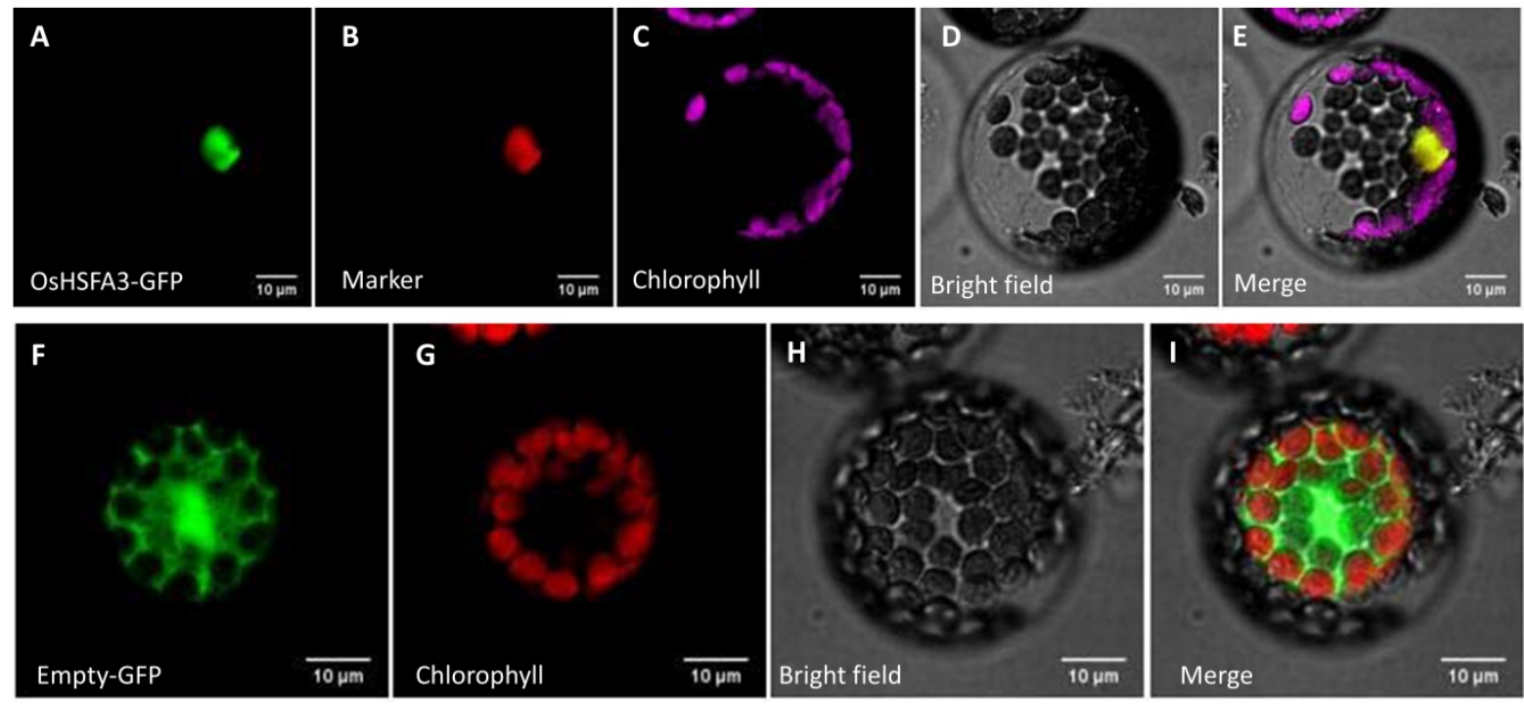

Figure 2. Subcellular localization of OsHSFA3 protein. Arabidopsis protoplasts were transiently transformed with constructs containing either OsHSFA3 fused with green fluorescent protein (GFP) (A-E) or GFP alone (F-I).

\subsection{Overexpression of OsHSFA3 Increases Osmotic and Drought Stress Tolerance in Transgenic Arabidopsis}

Given that OsHSFA3 showed a strong induction under drought stress, we speculated that OSHSFA3 may play an important role in drought stress response. To test our hypothesis, we overexpressed OsHSFA3 in Arabidopsis using CaMV 35S promoter. The overexpression vector was transformed into Arabidopsis ecotype Col-0 cv. by the floral dip method using Agrobacterium tumefaciens strain LBA4404. Of the total 11 positive transgenic lines, three independent lines expressing the transgene (designated as L1, L2 and L3 hereafter) were selected for further experiments along with the wild type (WT), i.e., Col-0 (Figure S3). 
We first imposed osmotic stresses to WT and transgenic Arabidopsis plants by supplementing $150 \mathrm{mM}$ mannitol to 10-day-old seedlings for 10 days and analyzed the root lengths (Figure 3A). Overall, the root growth was reduced in the presence of mannitol. However, significantly longer root lengths were observed in transgenic lines as compared to WT seedlings (Figure 3B). We also treated 10-day-old seedlings with $1 \mu \mathrm{M} \mathrm{ABA}$ and observed that root length reduced significantly in ABA-treated seedlings as compared to the seedlings treated as control. An important observation was the significant reduction of root length in all transgenic lines as compared to WT when treated with $\mathrm{ABA}$ (Figure 3B). These findings suggested that OsHSFA3 is responsive to osmotic stress and ABA.
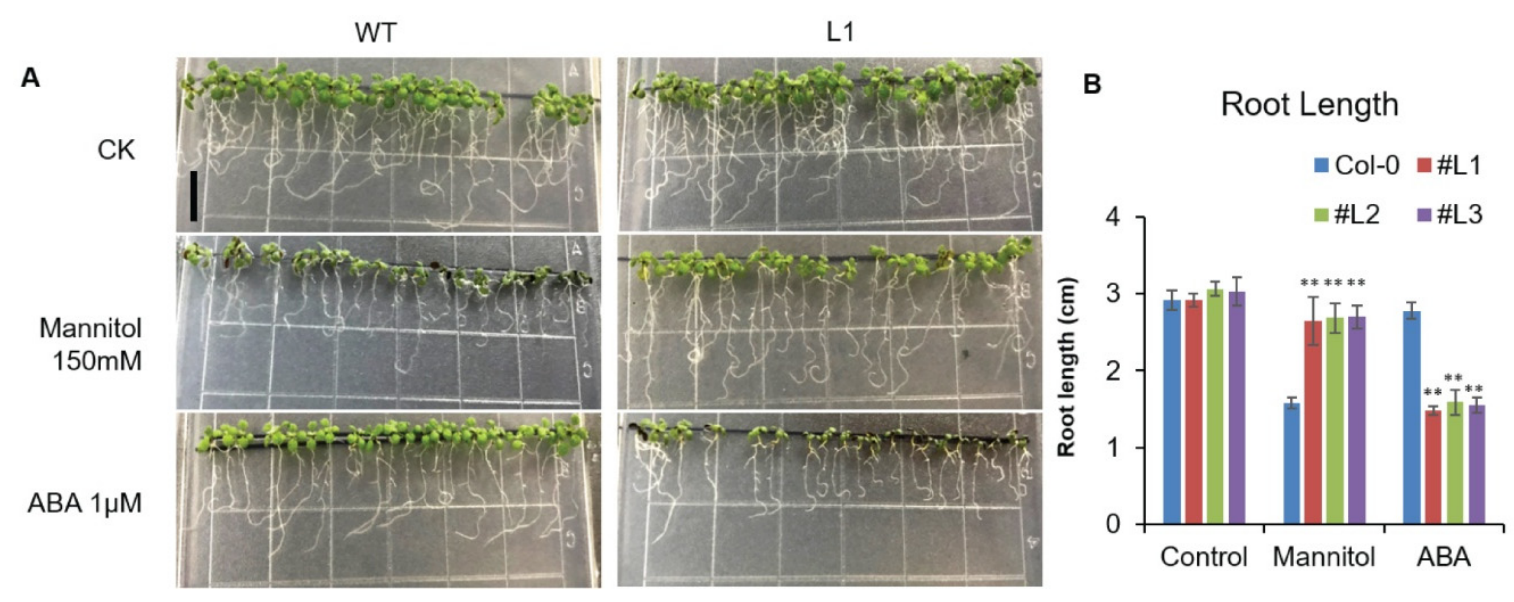

Figure 3. Root length observation of wild type (WT) and OsHSFA3-overexpressing transgenic Arabidopsis plants with mannitol and abscisic acid (ABA) treatment. (A) Seedlings of wild type and OsHSFA3 transgenic lines grew on MS medium in control (CK), mannitol treatment (150 mM) and ABA treatment $(1 \mu \mathrm{M})$. (B) Statistical analysis of root length of wild type and OsHSFA3-overexpressing Arabidopsis plants with and without mannitol and ABA treatment. Values indicate mean \pm SE of 10 biologically independent samples. Significance of data is tested with Student's $t$ test. ${ }^{* *}$ represents $p<0.01$.

Following osmotic stress, one-month-old plants were submitted to drought stress for 20 days by terminating watering (Figure 4A). Thereafter, seedlings were transferred to normal growth conditions (watering was resumed) and grown for five days. The survival rate of WT plants was $26 \%$, while an average of $83.3 \%$ of transgenic plants recovered (Figure 4B). Similarly, a decrease in biomass was observed under drought stress in all plants. However, the transgenic lines showed significantly higher biomass as compared to WT under drought stress (Figure 4C). Fresh weight is an important indicator of stress tolerance in plants. There was no significant difference in fresh weight among WT and transgenic plants under control conditions (Figure 4D). Nevertheless, a significantly higher fresh weight (FW) was observed in all transgenic lines as compared to WT under drought stress (Figure 4D). Water loss rates of detached leaves have been widely used to reflect drought tolerance in plants [18]. Water loss rates were assessed after every $30 \mathrm{~min}$ for both transgenic plants and control plants. We witnessed a steady leaf water loss with increasing time after dehydration in all plants. Notably, the water loss rate was higher in WT as compared to overexpressing transgenic lines (Figure 4E). These findings demonstrate that the transgenic lines were more resistant to dehydration and were drought stress tolerant. 




Figure 4. Assay of drought tolerance and associated traits for OsHSFA3 overexpression transgenic Arabidopsis plants. (A) Phenotypic analysis of one-month old WT and OsHSFA3 overexpression transgenic Arabidopsis plants stressed for 20 days. (B) Survival rate of plants shown in (A) after rewatering. (C,D) Plant biomass and seedling fresh weight under control and drought stress for WT and OsHSFA3-overexpressing transgenic Arabidopsis plants. (E) Water loss rate of the detached leaves of OsHSFA3-overexpressing transgenic lines and WT. Values indicate mean \pm SE of 10 biologically independent samples. Significance of data is tested with Student's $t$ test. ${ }^{* *}$ represents $p<0.01$.

\subsection{Overexpression of OsHSFA3 Increases Drought Tolerance by Decreasing $\mathrm{H}_{2} \mathrm{O}_{2}$ Accumulation and} Increasing ABA Levels

ROS accumulation is a common response under abiotic stresses which causes oxidative stress to plants [14]. Considering its vital role in drought tolerance, we investigated $\mathrm{H}_{2} \mathrm{O}_{2}$ levels in the WT and OsHSFA3-overexpressing lines. After 20 days under drought stress conditions, we witnessed an increase in $\mathrm{H}_{2} \mathrm{O}_{2}$ levels in WT and transgenic lines as compared to control conditions (Figure 5A). Significantly higher $\mathrm{H}_{2} \mathrm{O}_{2}$ levels were recorded in WT plants relative to transgenic plants (Figure $5 \mathrm{~A}$ ). Among transgenic lines, L2 and L3 had higher levels while L1 had slightly lower $\mathrm{H}_{2} \mathrm{O}_{2}$ levels. These results showed that WT plants accumulated more $\mathrm{H}_{2} \mathrm{O}_{2}$ as compared to transgenic lines under drought stress and thus were more sensitive to oxidative stress. Membrane lipid peroxidation is an important stress biomarker under different abiotic stresses. $\mathrm{H}_{2} \mathrm{O}_{2}$ accumulation normally enhances lipid peroxidation as a result of oxidative damage to cells [37]. Membrane lipid peroxidation is measured in terms of malondialdehyde (MDA). In this study, we observed a significant increase in MDA levels under drought stress in the WT as compared to transgenic plants (Figure 5B). These results suggested that OsHSFA3-overexpressing plants had maintained ROS homeostasis and thus had lower oxidative damage. 
A

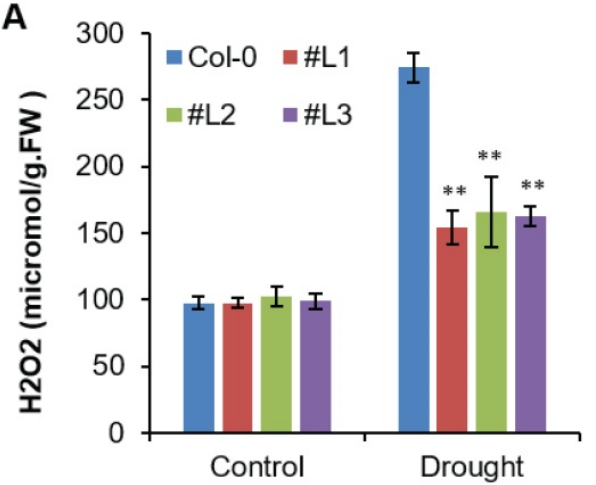

C
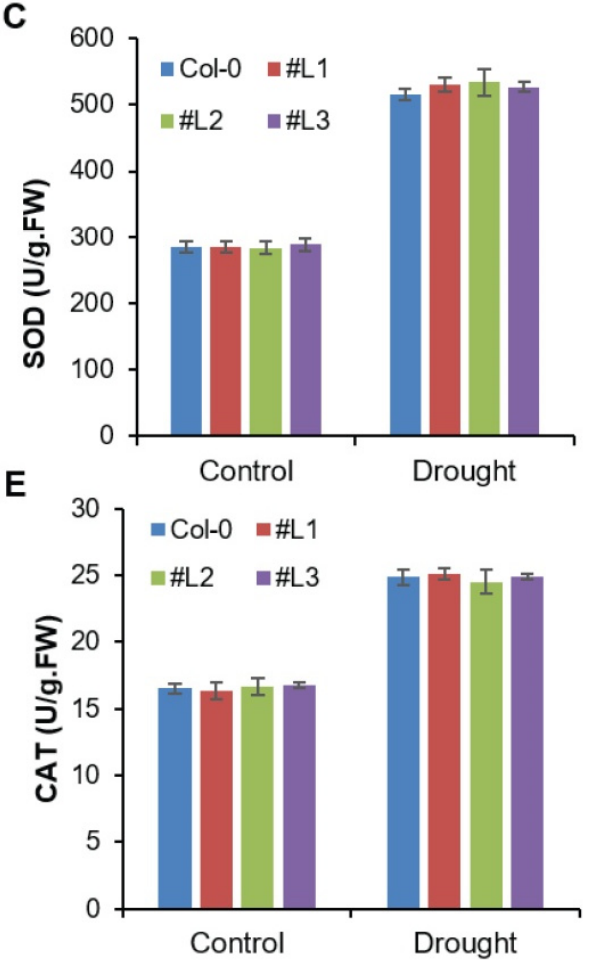

B

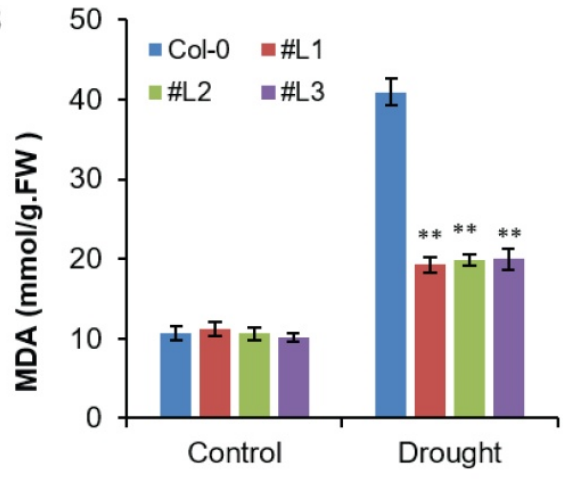

D
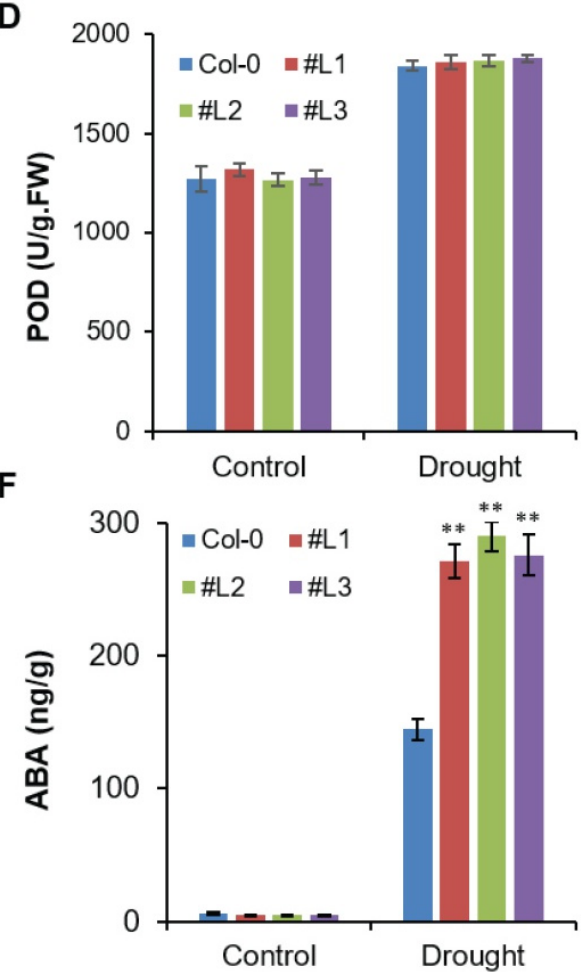

Figure 5. Reactive oxygen species (ROS) and antioxidant response under drought stress in OsHSFA3-overexpressing transgenic Arabidopsis plants. Quantitative measurement of $\mathrm{H}_{2} \mathrm{O}_{2}$ (A), malondialdehyde (B), superoxide dismutase (SOD) (C), peroxidase (POD) (D), catalase (CAT) (E) and ABA (F) in WT and OsHSFA3-overexpressing transgenic Arabidopsis plants under control and drought stress. Values indicate mean \pm SE of 10 biologically independent samples. Significance of data is tested with Student's $t$ test. ${ }^{* *}$ represents $p<0.01$.

Enzymatic antioxidants play important roles in ROS scavenging for normal plant growth $[13,38]$. To estimate the ability of OsHSFA3-overexpressing Arabidopsis lines and WT to scavenge ROS, the activities of antioxidant enzymes, i.e., SOD, POD and CAT, were measured. The activities of the three antioxidants were not apparently different between transgenic lines and WT plants under normal conditions. Observably, the response of transgenic and WT plants under drought was higher in terms of enzyme activities. However, no obvious differences were observed between WT and transgenic lines. These results suggest the overexpression of OsHSFA3 did not significantly modify the scavenging ability of transgenic Arabidopsis plants as compared to WT (Figure 5C-E).

ABA is an important plant stress signaling hormone which plays important role in drought stress tolerance via regulating expression of various stress-responsive genes [31,39,40]. Thus, we measured endogenous ABA levels in OsHSFA3-overexpressing lines and WT plants. ABA levels were significantly 
higher in drought-stressed plants as compared to control plants (Figure 5F). ABA content was higher in all transgenic lines than in WT plants after they were subjected to drought conditions. Under normal conditions, the ABA levels were very low and were not significantly different between WT and transgenic lines (Figure 5F). These results suggest that OsHSFA3 may play an important role in ABA signaling. These findings also correlate with the results of exogenous application of ABA to transgenic and WT seedlings (Figure 3B).

\subsection{OsHSFA3 Regulates Polyamines Biosynthesis under Drought Stress}

Further work was carried out to elucidate the molecular mechanism underlying the enhanced drought tolerance rendered by OSHSFA3. Commonly, plants would modulate a battery of functional genes and TFs, including HSFs, which are involved in the synthesis of various defense metabolites to resist the abiotic stress. To validate whether HSFs were involved in defense metabolite synthesis, we determined the accumulation of polyamines, i.e., diamine putrescine (Put), triamine spermidine (Spd) and tetraminespermine (Spm) [18]. We attempted to analyze whether polyamine synthesis was altered in the transgenic plants. In response to drought stress, Put levels were significantly increased in transgenic plants as compared to WT. Under controlled conditions, the levels were lower but still a difference between WT and transgenic lines existed (Figure 6A). Regarding Spd, we observed an increasing trend under drought stress as compared to control and a significant difference between WT and transgenic lines under both control and drought stress conditions was observed (Figure 6B). Notably, Spd levels were significantly higher in overexpressing lines as compared to WT, which suggested its putative role in drought tolerance. In the case of Spm, drought treatment led towards no significant changes in levels of Spm (Figure 6C). However, transgenic overexpressing lines still had significantly higher Spm levels as compared to WT (Figure 6C). These results concluded that OsHSFA3 regulates polyamines biosynthesis particularly under drought stress conditions, and that polyamines may play important roles in drought tolerance of the transgenic Arabidopsis plants overexpressing OsHSFA3.

\subsection{Expression of Polyamine Biosynthesis Genes is Induced under Drought Stress}

The key enzymes which regulate the polyamine biosynthesis include arginine decarboxylase (ADC), spermidine synthase (SPD) and spermine synthase(SPMS) [41]. In Arabidopsis thaliana, ADC production is regulated by two ADC paralogues, AtADC1 and AtADC2 [20]. Similarly, regulation of SPD and SPMS is controlled by the expression of the SPDS1 and SPMS genes, respectively [41]. We hypothesized that OsHSFA3 may regulate the expression of these key genes for drought tolerance. Thus, we analyzed the mRNA abundance levels of these genes under drought stress treatment in WT and OsHSFA3-overexpressing lines. The results showed that the expression of all tested genes was upregulated under drought conditions in both WT and transgenic plants, denoting that these genes are highly drought-responsive (Figure 6D-G). Notably, the mRNA abundance of the AtADC1 gene was significantly higher under drought in transgenic plants as compared to WT, indicating that AtADC1 could be directly influenced by OSHSFA3.

\subsection{OsHSFA3 Interacts with OsADC1 and AtADC1 Promoter}

HSF transcription factors normally regulate the expression of various stress-responsive genes via binding to heat shock elements (HSEs) [23]. In silico cis-element analysis of the promoter of OsADC1 and AtADC1 showed that they contain HSE elements, so can be effectively bound by HSFs. To determine if OSHSFA3 is involved in the activation of drought stress signaling pathways, we performed a yeast one-hybrid assay. The interaction between prey and bait was observed according to the growth of the yeast transformants in a series of 10 -fold dilutions. The results showed that the full length OsHSFA3 could significantly bind to OsADC1 (LOC_Os08g33620) as well as to the AtADC1 promoter (Figure 6H). These results indicate that OsHSFA3 was able to activate the expression of OsADC1 and AtADC1 by binding related cis-elements. 

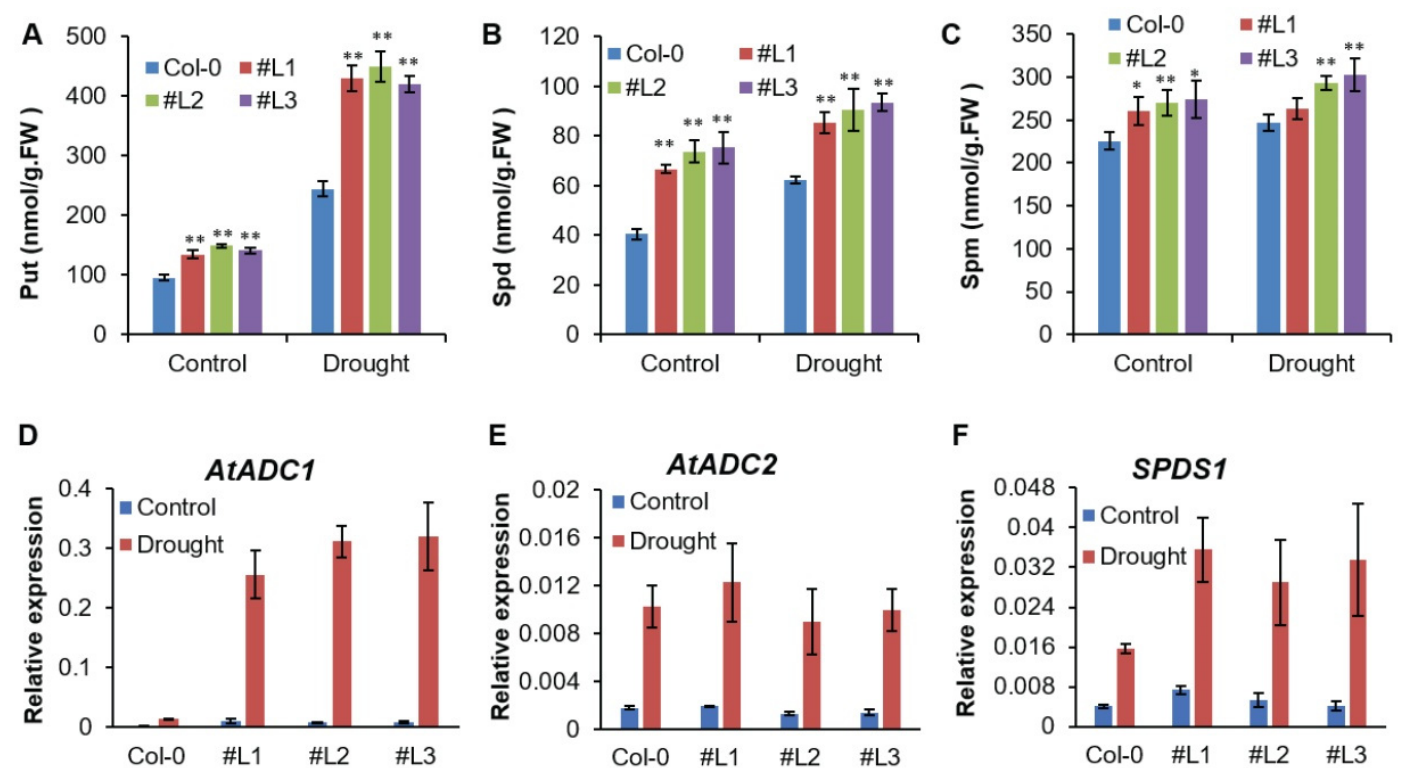

E

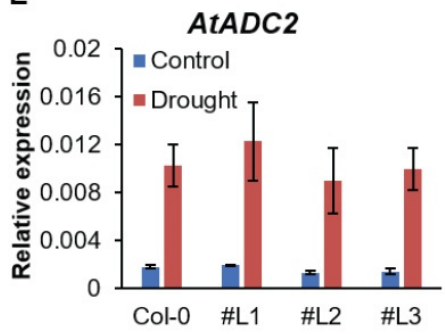

H

pGADT7-Rec2-OsHSFA3

pGADT7-Rec2-OsHSFA3

pGADT7-Rec2-OsHSFA3

pGADT7-Rec2-53
$\mathbf{F}$
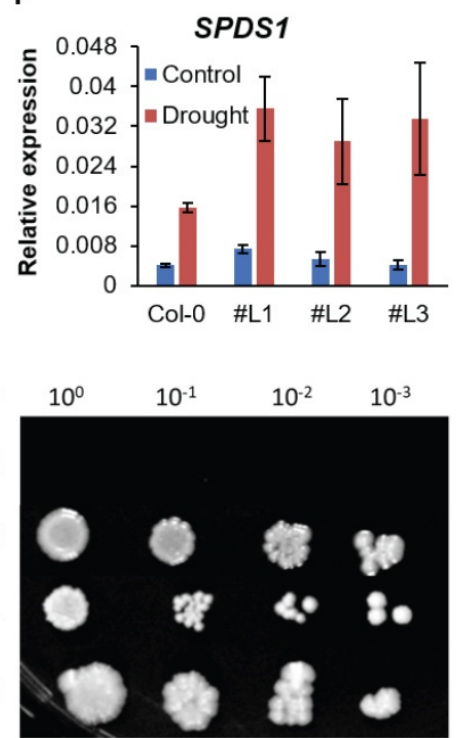

SD/-Leu/-Trp/-His 30 mM 3-AT

Figure 6. Analysis of polyamines, mRNA abundance and yeast one-hybrid assay for OsHSFA3. (A-C) Free polyamine contents in WT and OsHSFA3-overexpressing transgenic lines before and after drought stress. Put, putrescine; Spd, spermidine; Spm, spermine. Values indicate mean \pm SE of 3 biologically independent samples. Significance of data was tested with Student's $t$ test. * represents $p<0.05,{ }^{* *}$ represents $p<0.01$. (D-G) Quantitative real-time PCR analysis of expression levels of stress-responsive genes in WT and OsHSFA3-overexpressing transgenic plants under normal and drought conditions. (H) Yeast one-hybrid assay showing that OsHSFA3 can bind to AtADC1 and $O S A D C$ in vitro on a triple-dropout medium.

\section{Discussion}

Heat shock transcription factors (HSFs) constitute a large TF family with evident roles in growth, development and reproduction [36,42]. In addition, their important role in abiotic stress tolerance has emerged recently in several crop species [23,24,34,43]. HSFs are comprised of 25 members in rice and 22 members in Arabidopsis [28]. Although several members of rice HSFs have been reported for their role in growth and stress responses, the function and mechanism of action of many HSF genes are yet to be studied. The rice HSF TF OSHSFA3 has been shown to be responsive to cold and drought stress in rice $[35,36]$. However, how OsHSFA3 regulates drought tolerance in rice is not known yet. In this study, we confirmed that OsHSFA3 is induced upon drought treatment and the transcript level continued to increase with the duration of stress level, suggesting that OsHSFA3 may regulate drought tolerance in rice (Figure 1). Phylogenetic analysis with the orthologue genes indicated a close similarity with other monocots, particularly Setariaitalica, Zea mays and Sorghum bicolor. To further see the typical feature of a TF, we observed its subcellular localization in Arabidopsis protoplasts using GFP-fused protein. OsHSFA3 has shown nuclear localization, which was in accordance with other HSF TFs [34,44]. 
Arabidopsis serves as an excellent model plant to study the function of important genes belonging to different crop species, especially for abiotic stresses $[31,34,41,45,46]$. We thus overexpressed OsHSFA3 in Arabidopsis thaliana to study its response to drought stress. Root is an important agronomic trait that helps plants survive under limiting water conditions. Plants adjust their root length under drought conditions to cope with the water deficiency. We observed significant increases in the root length under mannitol treatment in transgenic Arabidopsis plants overexpressing OsHSFA3 as compared to WT (Figure 3), suggesting that OsHSFA3 may positively regulate drought tolerance. ABA is an important phytohormone that acts as a signaling molecule to activate the expression of various TFs under abiotic stress [47-49]. We observed a significant decrease in root length under ABA treatment in OsHSFA3-overexpressing plants as compared to WT, suggesting that OsHSFA3 is hypersensitive to ABA and ABA may regulate the expression of OsHSFA3 (Figure 3). Consistently, we observed higher ABA levels under drought stress in OsHSFA3-overexpressing plants as compared to WT (Figure 5), denoting that OsHSFA3 may work in the ABA-dependent pathway.

The most important feature showing the drought tolerance level in plants is the survival ability under drought stress $[47,50,51]$. We observed a significantly higher survival ability under drought in the OsHSFA3-overexpressing plants as compared to WT, suggesting an important role of OSHSFA3 in drought tolerance. Similarly, OsHSFA3-overexpressing plants maintained higher biomass as compared to WT under drought stress, which further revealed its role in drought tolerance. These observations were in line with previous findings which revealed higher plant biomass and survival ability in drought-tolerant plants $[40,52,53]$. These findings indicate that OsHSFA3 induces drought tolerance in transgenic plants by different plant adaptations such as longer root length, reduced water loss and modulating the ABA level to protect from dehydration.

Under different abiotic stresses including drought, the production of ROS is increased many fold, which leads to oxidative stress in plants $[1,30]$. Higher ROS levels induce lipid peroxidation in plants and cause injury to cell membranes [10,15]. In this study, we observed a sharp increase in $\mathrm{H}_{2} \mathrm{O}_{2}$ and MDA levels under drought stress in WT plants, but $\mathrm{H}_{2} \mathrm{O}_{2}$ and MDA levels were significantly less in OsHSFA3-overexpressing plants (Figure 5). This indicates a putative role of OsHSFA3 in ROS scavenging and drought tolerance. Plants normally protect themselves from oxidative stress by activating their antioxidant defense systems [14,30]. SOD, CAT and POD are the most common antioxidant enzymes which scavenge ROS by detoxifying $\mathrm{H}_{2} \mathrm{O}_{2}$ and $\mathrm{O}_{2}{ }^{-}$into water and stable oxygen [30]. Thus, these antioxidant enzymes play a key role in stress tolerance by ROS scavenging [1,54]. Although we observed a sharp increase in the activities of these antioxidant enzymes under drought stress, there was no significant difference in their activities among WT and OsHSFA3-overexpressing plants (Figure 5). This suggests that OsHSFA3 does not regulate the activities of antioxidant enzymes, and that OsHSFA3 plays a role in ROS scavenging by some other mechanism [16]. Further, we investigated only three key antioxidant enzymes (SOD, POD and CAT), so the possibility that other antioxidants not studied here were activated could not excluded.

In plants, PAs were found to play crucial roles not only in plant growth, physiology and reproduction, but also in conferring abiotic stress (salt, drought and metal/metalloid toxicity) tolerance by enhancing antioxidant defense and oxidative stress tolerance [21,41,55]. In this study, we observed a significant increase of PA content in OsHSFA3-overexpressing plants than in WT under both control as well as drought stress conditions (Figure 6). This increased PA level was correlated with increased expression of $A t A D C 1, A t A D C 2, S P D S 1$ and SPMS genes, which are the key genes regulating the PA level [19-21]. In addition, we showed that OsHSFA3 can bind to AtADC1 and OsADC via a yeast one-hybrid assay (Figure 6). Taken together, these findings demonstrated that OsHSFA3 improves drought tolerance in Arabidopsis by modulating polyamine levels to maintain ROS homeostasis. 


\section{Materials and Methods}

\subsection{Plant Growth Conditions and Stress Treatments}

Rice seedlings were grown in soil mixed with compost in a growth chamber at $28^{\circ} \mathrm{C}$ with $16 \mathrm{~h}$ light and $8 \mathrm{~h}$ dark. Drought stress was imposed to rice seedlings by withholding water for 7 days on 3-week-old plants. Control plants were regularly watered to ensure normal plant growth. Arabidopsis thaliana seeds were grown in $340 \mathrm{~mL}$ pots filled with a mixture of peat/forest soil and vermiculite $(3: 1)$ in a glasshouse at $21{ }^{\circ} \mathrm{C}$ with a light intensity of $50 \mu \mathrm{mol} \cdot \mathrm{m}^{-2} \cdot \mathrm{s}^{-1}$ and $70 \%$ relative humidity under $16 \mathrm{~h}$ light $/ 8 \mathrm{~h}$ dark conditions. Then, these seedlings were transferred into MS medium supplemented with 3\% (w/v) sucrose and $0.8 \%$ agar and were grown under the same growth conditions with Arabidopsis seeds. The plate-grown seedlings were transferred to soil after 1 week. For drought stress, 4-week-old plants were subjected to 20 days of drought stress by withholding water supply. Control (Ck) plants were regularly watered to ensure normal growth.

\subsection{Total RNA Isolation and $q R T-P C R$}

Total RNA from untreated and drought-treated plants was isolated using the RNA extraction kit (Tiangen, Beijing, China), then the first-strand cDNA was synthesized from 500 ng total RNA by reverse transcriptase (Invitrogen, Carlsbad, CA, USA). The cDNA was diluted ten times to use as template in qRT-PCR with SYBR Premix ExTaq Mix (Takara, Dalian, China). The procedures were followed as described earlier [35]. Primer sequences are mentioned in Table S1. The housekeeping actin gene was used as the reference gene.

\subsection{Gene structure Prediction and Phylogenetic Analysis}

Gene structure, including introns and exons of the OsHSFA3 gene, was investigated by using the online Gene Structure Display Server (http://gsds.cbi.pku.edu.cn/) as described earlier [23]. The genomic and coding sequences were retrieved from rice genome annotation project (http://rice.plantbiology. msu.edu/).

Homologue alignment was obtained using the online tool ExPASy bioinformatics resource portal (https://embnet.vital-it.ch/software/BOX_form.html). Amino acid sequences of all orthologs were retrieved from NCBI. Sequences were aligned using ClustalW and a phylogenetic tree was constructed using MEGA 7 software (Temple University, Philadelphia, PA, USA) using neighbor-joining method with 1000 bootstrap replicates.

\subsection{Vector Construction and Transformation}

To investigate the tolerance to drought and osmotic stress of OsHSFA3 transgenic lines after molecular identification, the vector overexpressing pCAMBIA 1301s (which is a modified form of the pCAMBIA1301 vector) was generated by inserting OsHSFA3 into Kpn I (5'-end) and BamH I ( $3^{\prime}$-end) sites, driven by the CaMV 35S promoter (Figure S1). The overexpressing vectors were then transformed to Arabidopsis ecotype col-0 cv by the floral dip method using Agrobacterium tumefaciens strain LBA4404. Eleven positive independent transgenic lines were obtained and three independent overexpression lines of Arabidopsis with high transcript levels of OsHSFA3 were selected for further experiments along with the control, i.e., Col-0.

\subsection{Subcellular Localization}

The subcellular localization was first predicted using the online bioinformatic tool, WoLF PSORT (www.genscript.com/tools/wolf-psort). For experimental validation, the OsHSFA3 coding sequence was fused with the GFP coding sequence into a pBWA(V)HS-GLosGFP vector and the nucleus marker vector (pBWA(V)HS-Nucleus-mKate) was cotransformed into Arabidopsis protoplasts. The transient expression of OsHSFA3-GFP was observed in Arabidopsis protoplasts under a confocal laser microscope. 
For the negative control, the empty vector containing only the GFP gene was also transformed into Arabidopsis protoplasts.

\subsection{Measurement of ROS, MDA and Antioxidant Activities}

ROS were measured quantitatively in terms of $\mathrm{H}_{2} \mathrm{O}_{2}$ as described earlier [13]. Briefly, $100 \mathrm{mg}$ of fresh tissue was harvested and grounded with liquid nitrogen. The fine powder was immersed with $1 \mathrm{~mL}$ buffer of $50 \mathrm{mM}$ sodium phosphate ( $\mathrm{pH}$ 7.4) for $20 \mathrm{~min}$ on ice. After centrifuging for $15 \mathrm{~min}$ at $12,000 \times \mathrm{g}$ and $4{ }^{\circ} \mathrm{C}, \mathrm{H}_{2} \mathrm{O}_{2}$ in the supernatant was quantified using spectrophotometer. $\mathrm{H}_{2} \mathrm{O}_{2}$, MDA, SOD, POD and CAT were assayed by using specific kits, respectively (Nanjing Jiancheng bioengineering Institute, Nanjing, China).

\subsection{Analysis of Polyamines and $A B A$}

PAs were assayed by high-performance liquid chromatography (HPLC) using the protocol described before [56]. PA showed a maximum peak at $254 \mathrm{~nm}$ by detecting with a UV detector. Soluble conjugated PA were calculated by subtracting the free PA from the acid-soluble PA. ABA was measured using the gas chromatography/mass spectrometry (GC-MS) method as described by Okamoto et al. [57].

\subsection{Yeast One Hybrid Assay}

Putative HSE cis-elements were identified in the promoter region of OsADC (LOC_Os08g33620) and AtADC1(At2g16500). Yeast one-hybrid assay was performed to investigate whether OsHSFA3 could bind with these HSE cis-elements in the promoters of OsADC and AtADC1. The full-length ORF of OsHSFA3 was amplified by PCR using gene-specific primers (Table S1) and cloned into pGADT7-Rec (Clontech, Mountain View, CA, USA) to create a prey vector (pGADT7-OsHSFA3). About $2000 \mathrm{bp}$ promoter sequences of $O S A D C$ and $A t A D C 1$ genes were respectively cloned into the bait vector. The OsHSFA3 prey vector was cotransformed with either the OsADC or AtADC1 bait vector into yeast strain Y187. The cotransformed yeasts containing the bait and prey were cultivated on the $\mathrm{SD} /$-Leu/-Trp/-His selective media supplemented with 0 or $3 \mathrm{mM} 3$-amino-1,2,4-triazole (3-AT) for 3 days according to the instructions for the Matchmaker ${ }^{\mathrm{TM}}$ Gold Yeast One Hybrid System (Clontech, USA). Yeasts cotransformed with pGADT7-Rec2-53 (the pGADT7-Rec2-53 plasmid containing a murine $p 53$ fused to GAL4 AD domain) and p53HIS2 (the p53HIS2 harboring three tandem copies of p53-binding DNA elements) were used as the positive control. The negative control was pGADT7-Rec2-OsHSFA3 and p53HIS2 cotransformation. The interaction between prey and bait was observed according to the growth of the yeast transformants in a series of 10 -fold dilutions.

\section{Conclusions}

In summary, this study revealed the role of rice OSHSFA3 as a positive regulator of drought tolerance [2]. Collectively, the results indicate that OsHSFA3 works in an ABA-dependent pathway to limit water loss and maintains ROS homeostasis via regulating PA levels and the expression of various PA biosynthesis genes (Figure S4). Further studies could unravel more downstream targets of OsHSFA3 to have a deeper understanding of the drought signaling network

Supplementary Materials: The following are available online http://www.mdpi.com/1422-0067/21/5/1857/s1. Figure S1, map of the vector used for the transformation of Arabidopsis thaliana. Figure S2, conserved domains and gene structure of OSHSFA3. Figure S3, identification of positive Arabidopsis overexpressing lines. Figure S4, proposed working model for OsHSFA3. Table S1, primer sequences used in the study.

Author Contributions: Conceptualization, M.-D.Z., M.Z., Y.-M.L. and B.Z.; methodology, D.-J.G., S.-J.T. and K.Z.; software, K.Z.; formal analysis, D.-J.G. and S.-J.T.; investigation, D.-J.G. and S.T.; resources, D.-J.G. and S.-J.T.; data curation, S.-J.T.; writing-original draft preparation, M.-D.Z., D.-J.G. and S.-J.T.; writing-review and editing, M.Z, Y.-M.L. and B.Z.; visualization, D.-J.G.; supervision, Y.-M.L. and B.Z.; funding acquisition, Y.-M.L. and B.Z. All authors have read and agreed to the published version of the manuscript. 
Funding: This work was funded by the National Key R\&D Program of China (2018YFD0301001) and National Natural Science Foundation of Hunan Province (2019JJ50332).

Conflicts of Interest: The authors declare no conflict of interest.

\section{References}

1. Hussain, H.A.; Men, S.; Hussain, S.; Chen, Y.; Ali, S.; Zhang, S.; Zhang, K.; Li, Y.; Xu, Q.; Liao, C.; et al. Interactive effects of drought and heat stresses on morpho-physiological attributes, yield, nutrient uptake and oxidative status in maize hybrids. Sci. Rep. 2019, 9, 3890. [CrossRef]

2. Zafar, S.A.; Zaidi, S.S.-e.-A.; Gaba, Y.; Singla-Pareek, S.L.; Dhankher, O.P.; Li, X.; Mansoor, S.; Pareek, A. Engineering Abiotic Stress Tolerance via CRISPR-Cas mediated genome editing. J. Exp. Bot. 2019, 71, 470-479. [CrossRef]

3. Saeed, Z.; Naveed, M.; Imran, M.; Bashir, M.A.; Sattar, A.; Mustafa, A.; Hussain, A.; Xu, M. Combined use of Enterobacter sp. MN17 and zeolite reverts the adverse effects of cadmium on growth, physiology and antioxidant activity of Brassica napus. PLoS ONE 2019, 14, e0213016. [CrossRef]

4. Arshad, M.S.; Farooq, M.; Asch, F.; Krishna, J.S.; Prasad, P.V.; Siddique, K.H. Thermal stress impacts reproductive development and grain yield in rice. Plant Physiol. Biochem. 2017, 115, 57-72. [CrossRef] [PubMed]

5. Zafar, S.A.; Noor, M.A.; Waqas, M.A.; Wang, X.; Shaheen, T.; Raza, M. Temperature extremes in cotton production and mitigation strategies. In Past, Present and Future Trends in Cotton Breeding; Rahman, M.R., Zafar, Y., Eds.; IntechOpen: London, UK, 2018; pp. 65-91.

6. Poli, Y.; Basava, R.K.; Panigrahy, M.; Vinukonda, V.P.; Dokula, N.R.; Voleti, S.R.; Desiraju, S.; Neelamraju, S. Characterization of a Nagina22 rice mutant for heat tolerance and mapping of yield traits. Rice 2013, 6, 36. [CrossRef] [PubMed]

7. Ahmadi, N.; Audebert, A.; Bennett, M.J.; Bishopp, A.; de Oliveira, A.C.; Courtois, B.; Diedhiou, A.; Diévart, A.; Gantet, P.; Ghesquière, A. The roots of future rice harvests. Rice 2014, 7, 29. [CrossRef] [PubMed]

8. Dubouzet, J.G.; Sakuma, Y.; Ito, Y.; Kasuga, M.; Dubouzet, E.G.; Miura, S.; Seki, M.; Shinozaki, K.; Yamaguchi-Shinozaki, K. OsDREB genes in rice, Oryza sativa L., encode transcription activators that function in drought, high salt and cold responsive gene expression. Plant J. 2003, 33, 751-763. [CrossRef] [PubMed]

9. Das, P.; Lakra, N.; Nutan, K.K.; Singla-Pareek, S.L.; Pareek, A. A unique bZIP transcription factor imparting multiple stress tolerance in Rice. Rice 2019, 12, 58. [CrossRef] [PubMed]

10. Hussain, H.A.; Hussain, S.; Khaliq, A.; Ashraf, U.; Anjum, S.A.; Men, S.; Wang, L. Chilling and Drought Stresses in Crop Plants: Implications, Cross Talk, and Potential Management Opportunities. Front. Plant Sci. 2018, 9, 393. [CrossRef]

11. Ribeiro, C.W.; Korbes, A.P.; Garighan, J.A.; Jardim-Messeder, D.; Carvalho, F.E.L.; Sousa, R.H.V.; Caverzan, A.; Teixeira, F.K.; Silveira, J.A.G.; Margis-Pinheiro, M. Rice peroxisomal ascorbate peroxidase knockdown affects ROS signaling and triggers early leaf senescence. Plant Sci. 2017, 263, 55-65. [CrossRef]

12. Ashraf, M. Inducing drought tolerance in plants: Recent advances. Biotechnol. Adv. 2010, 28, 169-183. [CrossRef] [PubMed]

13. Zafar, S.A.; Patil, S.B.; Uzair, M.; Fang, J.; Zhao, J.; Guo, T.; Yuan, S.; Uzair, M.; Luo, Q.; Shi, J.; et al. DEGENERATED PANICLE AND PARTIAL STERILITY 1 (DPS1) encodes a cystathionine beta-synthase domain containing protein required for anther cuticle and panicle development in rice. New Phytol. 2020, 225, 356-375. [CrossRef] [PubMed]

14. Zafar, S.A.; Hameed, A.; Nawaz, M.A.; Wei, M.; Noor, M.A. Mechanisms and molecular approaches for heat tolerance in rice (Oryza sativa L.) under climate change scenario. J. Integr. Agric. 2018, 17, 726-738. [CrossRef]

15. Zhao, Q.; Zhou, L.; Liu, J.; Du, X.; Asad, M.A.; Huang, F.; Pan, G.; Cheng, F. Relationship of ROS accumulation and superoxide dismutase isozymes in developing anther with floret fertility of rice under heat stress. Plant Physiol. Biochem. 2018, 122, 90-101. [CrossRef] [PubMed]

16. Hu, L.; Liang, W.; Yin, C.; Cui, X.; Zong, J.; Wang, X.; Hu, J.; Zhang, D. Rice MADS3 regulates ROS homeostasis during late anther development. Plant Cell 2011, 23, 515-533. [CrossRef] [PubMed] 
17. Alcázar, R.; Bitrián, M.; Bartels, D.; Koncz, C.; Altabella, T.; Tiburcio, A.F. Polyamine metabolic canalization in response to drought stress in Arabidopsis and the resurrection plant Craterostigma plantagineum. Plant Signal. Behav. 2011, 6, 243-250. [CrossRef] [PubMed]

18. Li, K.; Xing, C.; Yao, Z.; Huang, X. PbrMYB21, a novel MYB protein of Pyrus betulaefolia, functions in drought tolerance and modulates polyamine levels by regulating arginine decarboxylase gene. Plant Biotechnol. J. 2017, 15, 1186-1203. [CrossRef]

19. Sánchez-Rangel, D.; Chávez-Martínez, A.I.; Rodríguez-Hernández, A.A.; Maruri-López, I.; Urano, K.; Shinozaki, K.; Jiménez-Bremont, J.F. Simultaneous Silencing of Two Arginine Decarboxylase Genes Alters Development in Arabidopsis. Front. Plant Sci. 2016, 7, 300. [CrossRef]

20. Maruri-López, I.; Jiménez-Bremont, J.F. Hetero-and homodimerization of Arabidopsis thaliana arginine decarboxylase AtADC1 and AtADC2. Biochem. Biophys. Res. Commun. 2017, 484, 508-513. [CrossRef]

21. Do, P.T.; Drechsel, O.; Heyer, A.G.; Hincha, D.K.; Zuther, E. Changes in free polyamine levels, expression of polyamine biosynthesis genes, and performance of rice cultivars under salt stress: A comparison with responses to drought. Front. Plant Sci. 2014, 5, 182. [CrossRef]

22. Liu, T.; Wook Kim, D.; Niitsu, M.; Berberich, T.; Kusano, T. POLYAMINE OXIDASE 1 from rice (Oryza sativa) is a functional ortholog of Arabidopsis POLYAMINE OXIDASE 5. Plant Signal. Behav. 2014, 9, e29773. [CrossRef] [PubMed]

23. Zafar, S.A.; Hussain, M.; Raza, M.; Ahmed, H.G.M.-D.; Rana, I.A.; Sadia, B.; Atif, R.M. Genome wide analysis of heat shock transcription factor (HSF) family in chickpea and its comparison with Arabidopsis. Plant Omics 2016, 9, 136-141. [CrossRef]

24. Li, P.-S.; Yu, T.-F.; He, G.-H.; Chen, M.; Zhou, Y.-B.; Chai, S.-C.; Xu, Z.-S.; Ma, Y.-Z. Genome-wide analysis of the Hsf family in soybean and functional identification of GmHsf-34 involvement in drought and heat stresses. BMC Genom. 2014, 15, 1009. [CrossRef] [PubMed]

25. Ali, M.A.; Azeem, F.; Nawaz, M.A.; Acet, T.; Abbas, A.; Imran, Q.M.; Shah, K.H.; Rehman, H.M.; Chung, G.; Yang, S.H.; et al. Transcription factors WRKY11 and WRKY17 are involved in abiotic stress responses in Arabidopsis. J. Plant Physiol. 2018, 226, 12-21. [CrossRef]

26. Wu, X.; Shiroto, Y.; Kishitani, S.; Ito, Y.; Toriyama, K. Enhanced heat and drought tolerance in transgenic rice seedlings overexpressing OsWRKY11 under the control of HSP101 promoter. Plant Cell Rep. 2009, 28, 21-30. [CrossRef] [PubMed]

27. Wan, L.; Zhang, J.; Zhang, H.; Zhang, Z.; Quan, R.; Zhou, S.; Huang, R. Transcriptional activation of OsDERF1 in OsERF3 and OsAP2-39 negatively modulates ethylene synthesis and drought tolerance in rice. PLoS ONE 2011, 6, e25216. [CrossRef]

28. Guo, J.; Wu, J.; Ji, Q.; Wang, C.; Luo, L.; Yuan, Y.; Wang, Y.; Wang, J. Genome-wide analysis of heat shock transcription factor families in rice and Arabidopsis. J. Genet. Genom. 2008, 35, 105-118. [CrossRef]

29. Wang, C.; Zhang, Q.; Shou, H.-x. Identification and expression analysis of OsHsfs in rice. J. Zhejiang Univ. Sci. B 2009, 10, 291-300. [CrossRef]

30. Mittler, R. Oxidative stress, antioxidants and stress tolerance. Trends Plant Sci. 2002, 7, 405-410. [CrossRef]

31. Zhao, J.; Zhao, L.; Zhang, M.; Zafar, S.A.; Fang, J.; Li, M.; Zhang, W.; Li, X. Arabidopsis E3 Ubiquitin Ligases PUB22 and PUB23 Negatively Regulate Drought Tolerance by Targeting ABA Receptor PYL9 for Degradation. Int. J. Mol. Sci. 2017, 18, 1841. [CrossRef]

32. Dang, J.; Jiang, M.; Lin, F. ABA up-regulate the expression of OsHsf gene in leaves of rice plants. J. Nanjing Agric. Univ. 2010, 33, 11-15.

33. Schmidt, R.; Schippers, J.H.; Welker, A.; Mieulet, D.; Guiderdoni, E.; Mueller-Roeber, B. Transcription factor OsHsfC1b regulates salt tolerance and development in Oryza sativa ssp. japonica. AoB Plants 2012, 2012, pls011. [CrossRef]

34. Yokotani, N.; Ichikawa, T.; Kondou, Y.; Matsui, M.; Hirochika, H.; Iwabuchi, M.; Oda, K. Expression of rice heat stress transcription factor OsHsfA2e enhances tolerance to environmental stresses in transgenic Arabidopsis. Planta 2008, 227, 957-967. [CrossRef]

35. Lv, Y.; Xu, L.; Dossa, K.; Zhou, K.; Zhu, M.; Xie, H.; Tang, S.; Yu, Y.; Guo, X.; Zhou, B. Identification of putative drought-responsive genes in rice using gene co-expression analysis. Bioinformation 2019, 15, 480-489. [CrossRef] [PubMed] 
36. Chauhan, H.; Khurana, N.; Agarwal, P.; Khurana, P. Heat shock factors in rice (Oryza sativa L.): Genome-wide expression analysis during reproductive development and abiotic stress. Mol. Genet. Genom. 2011, 286, 171. [CrossRef] [PubMed]

37. Hameed, A.; Goher, M.; Iqbal, N. Heat stress-induced cell death, changes in antioxidants, lipid peroxidation, and protease activity in wheat leaves. J. Plant Growth Regul. 2012, 31, 283-291. [CrossRef]

38. Kamran, M.; Malik, Z.; Parveen, A.; Huang, L.; Riaz, M.; Bashir, S.; Mustafa, A.; Abbasi, G.H.; Xue, B.; Ali, U. Ameliorative Effects of Biochar on Rapeseed (Brassica napus L.) Growth and Heavy Metal Immobilization in Soil Irrigated with Untreated Wastewater. J. Plant Growth Regul. 2019. [CrossRef]

39. Lim, C.W.; Baek, W.; Jung, J.; Kim, J.-H.; Lee, S.C. Function of ABA in Stomatal Defense against Biotic and Drought Stresses. Int. J. Mol. Sci. 2015, 16, 15251-15270. [CrossRef]

40. Ma, Q.; Xia, Z.; Cai, Z.; Li, L.; Cheng, Y.; Liu, J.; Nian, H. GmWRKY16 Enhances Drought and Salt Tolerance Through an ABA-Mediated Pathway in Arabidopsis thaliana. Front. Plant Sci. 2019, 9, 1979. [CrossRef]

41. Kasukabe, Y.; He, L.; Nada, K.; Misawa, S.; Ihara, I.; Tachibana, S. Overexpression of Spermidine Synthase Enhances Tolerance to Multiple Environmental Stresses and Up-Regulates the Expression of Various Stress-Regulated Genes in Transgenic Arabidopsis thaliana. Plant Cell Physiol. 2004, 45, 712-722. [CrossRef]

42. Li, J.; Chauve, L.; Phelps, G.; Brielmann, R.M.; Morimoto, R.I. E2F coregulates an essential HSF developmental program that is distinct from the heat-shock response. Genes Dev. 2016, 30, 2062-2075. [CrossRef] [PubMed]

43. Chauhan, H.; Khurana, N.; Agarwal, P.; Khurana, J.P.; Khurana, P. A seed preferential heat shock transcription factor from wheat provides abiotic stress tolerance and yield enhancement in transgenic Arabidopsis under heat stress environment. PLoS ONE 2013, 8, e79577. [CrossRef] [PubMed]

44. Zhang, S.; Xu, Z.-S.; Li, P.; Yang, L.; Wei, Y.; Chen, M.; Li, L.; Zhang, G.; Ma, Y. Overexpression of TaHSF3 in Transgenic Arabidopsis Enhances Tolerance to Extreme Temperatures. Plant Mol. Biol. Report. 2013, 31, 688-697. [CrossRef]

45. Gu, L.; Ma, Q.; Zhang, C.; Wang, C.; Wei, H.; Wang, H.; Yu, S. The Cotton GhWRKY91 Transcription Factor Mediates Leaf Senescence and Responses to Drought Stress in Transgenic Arabidopsis thaliana. Front. Plant Sci. 2019, 10, 1352. [CrossRef] [PubMed]

46. Liu, A.-L.; Zou, J.; Liu, C.-F.; Zhou, X.-Y.; Zhang, X.-W.; Luo, G.-Y.; Chen, X.-B. Over-expression of OsHsfA7 enhanced salt and drought tolerance in transgenic rice. BMB Rep. 2013, 46, 31. [CrossRef] [PubMed]

47. Lu, P.; Magwanga, R.O.; Kirungu, J.N.; Hu, Y.; Dong, Q.; Cai, X.; Zhou, Z.; Wang, X.; Zhang, Z.; Hou, Y. Overexpression of Cotton a DTX/MATE Gene Enhances Drought, Salt and Cold Stress Tolerance in Transgenic Arabidopsis. Front. Plant Sci. 2019, 10, 299. [CrossRef]

48. Vishwakarma, K.; Upadhyay, N.; Kumar, N.; Yadav, G.; Singh, J.; Mishra, R.K.; Kumar, V.; Verma, R.; Upadhyay, R.; Pandey, M. Abscisic acid signaling and abiotic stress tolerance in plants: A review on current knowledge and future prospects. Front. Plant Sci. 2017, 8, 161. [CrossRef]

49. Li, H.-M.; Liu, S.-D.; Ge, C.-W.; Zhang, X.-M.; Zhang, S.-P.; Chen, J.; Shen, Q.; Ju, F.-Y.; Yang, Y.-F.; Li, Y.; et al. Analysis of Drought Tolerance and Associated Traits in Upland Cotton at the Seedling Stage. Int. J. Mol. Sci. 2019, 20, 3888. [CrossRef]

50. Wang, N.-N.; Xu, S.-W.; Sun, Y.-L.; Liu, D.; Zhou, L.; Li, Y.; Li, X.-B. The cotton WRKY transcription factor (GhWRKY33) reduces transgenic Arabidopsis resistance to drought stress. Sci. Rep. 2019, 9, 724. [CrossRef]

51. Wattoo, F.M.; Rana, R.M.; Fiaz, S.; Zafar, S.A.; Noor, M.A.; Hassan, H.M.; Bhatti, M.H.; Rehman, S.U.; Anis, G.B.; Amir, R.M. Identification of drought tolerant maize genotypes and seedling based morpho-physiological selection indices for crop improvement. Sains Malays. 2018, 47, 295-302.

52. Noman, M.; Jameel, A.; Qiang, W.-D.; Ahmad, N.; Liu, W.-C.; Wang, F.-W.; Li, H.-Y. Overexpression of GmCAMTA12 Enhanced Drought Tolerance in Arabidopsis and Soybean. Int. J. Mol. Sci. 2019, 20, 4849. [CrossRef] [PubMed]

53. Baek, D.; Kim, M.C.; Kumar, D.; Park, B.; Cheong, M.S.; Choi, W.; Park, H.C.; Chun, H.J.; Park, H.J.; Lee, S.Y.; et al. AtPR5K2, a PR5-Like Receptor Kinase, Modulates Plant Responses to Drought Stress by Phosphorylating Protein Phosphatase 2Cs. Front. Plant Sci. 2019, 10, 1146. [CrossRef] [PubMed]

54. Di Meo, S.; Napolitano, G.; Venditti, P. Physiological and Pathological Role of ROS: Benefits and Limitations of Antioxidant Treatment. Int. J. Mol. Sci. 2019, 20, 4810. [CrossRef] [PubMed]

55. Hasanuzzaman, M.; Alhaithloul, H.A.S.; Parvin, K.; Bhuyan, M.H.M.B.; Tanveer, M.; Mohsin, S.M.; Nahar, K.; Soliman, M.H.; Mahmud, J.A.; Fujita, M. Polyamine Action under Metal/Metalloid Stress: Regulation of Biosynthesis, Metabolism, and Molecular Interactions. Int. J. Mol. Sci. 2019, 20, 3215. [CrossRef] 
56. Hu, X.; Zhang, Y.; Shi, Y.; Zhang, Z.; Zou, Z.; Zhang, H.; Zhao, J. Effect of exogenous spermidine on polyamine content and metabolism in tomato exposed to salinity-alkalinity mixed stress. Plant Physiol. Biochem. 2012, 57, 200-209. [CrossRef]

57. Okamoto, M.; Hanada, A.; Kamiya, Y.; Yamaguchi, S.; Nambara, E. Measurement of abscisic acid and gibberellins by gas chromatography/mass spectrometry. Methods Mol. Biol. 2009, 495, 53-60. 